\title{
CT Scan Features and Clinical Course of COVID-19 Pneumonia in Pregnant Women: Can CT Predict the Prognosis?
}

\author{
Hassan Hashemi ${ }^{1,2}$, Behnaz Moradi ${ }^{1,3}$, Hoda Sharif ${ }^{4, *}$, Mohammad Ali Kazemi ${ }^{1,5}$ and Masoumeh Gity \\ (iD) 1,2 \\ ${ }^{1}$ Advanced Diagnostic and Interventional Radiology Research Center (ADIR), Tehran University of Medical Sciences, Tehran, Iran \\ ${ }^{2}$ Department of Radiology, Medical Imaging Center, Imam Khomeini Hospital Complex, Tehran University of Medical Sciences, Tehran, Iran \\ ${ }^{3}$ Yas Complex Hospital, Tehran University of Medical Sciences, Tehran, Iran \\ ${ }^{4}$ Department of Radiology, Ganjavian Hospital, Dezful University of Medical Sciences, Dezful, Iran \\ ${ }^{5}$ Department of Radiology, Amiralam Hospital, Tehran University of Medical Sciences, Tehran, Iran \\ "Corresponding author: Department of Radiology, Ganjavian Hospital, Dezful University of Medical Sciences, Dezful, Iran. Email: hodasharif24@yahoo.com
}

Received 2020 June 19; Revised 2020 July 15; Accepted 2020 October 18.

Keywords: COVID-19 Pneumonia, CT Scan Features, Pregnancy

\section{Dear Editor,}

Coronavirus (COVID-19) infection, a new highly contagious respiratory disease has raised a significant amount of concern throughout the world. As in any other infectious disease, some groups are at higher risk and need more attention including elderly people or those who suffer from comorbidities. Pregnancy by itself is considered a state of partial immune suppression because of physiological changes in immune and cardiopulmonary systems which make pregnant women vulnerable to viral infections and severe illness after respiratory virus infections (1). On the other hand according to data during previous epidemics of coronavirus infections (SARS-COV and MERS$\mathrm{COV}$ ), pregnant women were more prone to present with critical clinical features and perinatal outcomes (1). Although according to initial reports mostly from China, COVID-19 does not seem to follow such a critical course in pregnancy, maternal death can occur in patients with severe disease (2). Therefore, it seems reasonable to pay attention to pregnant women in this worldwide pandemic as well as comparing them with non-pregnant affected cases.

Most common clinical features in pregnant women have been reported as fever, cough, myalgia, malaise, sore throat, diarrhea, and shortness of breath, which are similar to non-pregnant women $(1,3)$. In comparing lab data, lymphopenia and increased C-reactive protein (CRP) levels were found in the majority of patients (1). Low dose computed tomography (CT) scan plays an important role in the diagnosis of COVID-19 pneumonia in pregnant women and its severity assessment $(1,3,4)$. It is reassuring that the radiation dose to the fetus is negligible (0.01-0.66 mGy) from a single chest CT scan, and exposure to radiation of $<50 \mathrm{mGy}$ is not associated with an increased risk of fetal anomalies or pregnancy loss (5). On the other hand, it has been shown that CT scan has a superior sensitivity compared to RT-PCR in the early detection of COVID-19 pneumonia (4).

There are limited studies that have focused on the pattern of CT involvement and the pattern of timeline progression in pregnant patients. Regardless of being or not being pregnant, the most common early chest CT findings were reported as ground glass opacities (GGO) mostly in the lower lobes with peripheral distribution. Imaging findings of COVID-19 have overlap with other viral infections, but the patterns of involvement with predominantly peripheral distribution will facilitate the diagnosis of COVID19 pneumonia (4). With progression of COVID-19 disease, CT scan showed crazy paving pattern and consolidations that were followed by gradual absorption $(3,4)$. It has been shown that the $\mathrm{CT}$ features, the timeline progression, and the CT severity scores at each stage of the disease were similar to non-pregnant women (3). Furthermore, Liu et al. found that consolidation was significantly more common in the infected pregnant groups compared to nonpregnant women, while GGO \pm reticulation was more frequent in the non-pregnant adults (4). Also, they showed that presence of more consolidation and relatively higher CT severity score in infected pregnant women suggested a more severe disease (4). The CT severity score is an index proposed for assessment of severity of lung involvement by COVID-19 pneumonia (4).

Findings about perinatal outcome are controversial. Some suggest no evidence of poor perinatal outcome (fe- 
tal death, neonatal death, neonatal asphyxia and low Apgar score) $(1,3)$. Whereas, others report challenging perinatal outcomes including preterm labor and fetal distress (6), coagulopathy accompanied by liver dysfunction (6), and mother's death $(2,6)$. There is a recently published article by Hantoushzadeh et al. on severely involved pregnant women with high maternal death rate [seven out of nine cases] that focused especially on their clinical course and laboratory findings but not on the severity of findings in chest CT scan (2). We think such critically ill mothers possibly have higher CT severity scores and assessing this index could easily guide clinicians to evaluate the situation of their patients. There is no doubt that chest CT is an efficient and easily available modality for early detection and monitoring of the clinical course of patients with COVID19 , but there is limited data about its role in predicting the severity of COVID-19 pneumonia and prognosis of the disease in pregnant women. We recommend large-scale studies to assess the role of CT in determining the severity of the disease in pregnant women, which would be very helpful along with laboratory data and clinical features to structure the clinical treatment strategy.

\section{Footnotes}

Authors' Contribution: Behnaz Moradi. developed the original idea and the protocol, abstracted and analyzed data and edited the manuscript. Hoda Sharif. abstracted data and prepared the manuscript. Moham-
madAli Kazemi, Hassan Hashemi, and Masoumeh Gity contributed to the development of the protocol.

Conflict of Interests: There is no personal financial interests.

Ethical Approval: Ethical approval was not necessary.

Funding/Support: There was no funding/support.

\section{References}

1. Yang $\mathrm{H}$, Wang $\mathrm{C}$, Poon LC. Novel coronavirus infection and pregnancy. Ultrasound Obstet Gynecol. 2020;55(4):435-7. doi: 10.1002/uog.22006. [PubMed: 32134165]. [PubMed Central: PMC7169856].

2. Hantoushzadeh S, Shamshirsaz AA, Aleyasin A, Seferovic MD, Aski SK, Arian SE, et al. Maternal death due to COVID-19. Am J Obstet Gynecol. 2020;223(1):109 e1-109 e16. doi: 10.1016/j.ajog.2020.04.030. [PubMed: 32360108]. [PubMed Central: PMC7187838].

3. Liu D, Li L, Wu X, Zheng D, Wang J, Liang B, et al. Pregnancy and Perinatal Outcomes of Women with COVID-19 Pneumonia: A Preliminary Analysis. SSRN Electronic Journal. 2020. doi:10.2139/ssrn.3548758.

4. Liu H, Liu F, Li J, Zhang T, Wang D, Lan W. Clinical and CT imaging features of the COVID-19 pneumonia: Focus on pregnant women and children. J Infect. 2020;80(5):e7-e13. doi: 10.1016/j.jinf.2020.03.007. [PubMed: 32171865]. [PubMed Central: PMC7156118].

5. Poon LC, Yang H, Dumont S, Lee JCS, Copel JA, Danneels L, et al. ISUOG Interim Guidance on coronavirus disease 2019 (COVID-19) during pregnancy and puerperium: information for healthcare professionals - an update. Ultrasound Obstet Gynecol. 2020;55(6):84862. doi: 10.1002/uog.22061. [PubMed: 32356590]. [PubMed Central: PMC7267438].

6. Panahi L, Amiri M, Pouy S. Risks of Novel Coronavirus Disease (COVID 19) in Pregnancy; a Narrative Review. Arch Acad Emerg Med. 2020;8(1). e34. [PubMed: 32232217]. [PubMed Central: PMC7092922]. 